\title{
PROJECT OF BOOSTER SYNCHROTRON FOR DUKE FEL STORAGE RING*
}

\author{
S.F.Mikhailov, V.N.Litvinenko, P.Morcombe, G.Swift, \\ FEL Laboratory, Duke University, Durham, NC 27708, USA \\ N.A.Vinokurov, N.G.Gavrilov, Yu.G.Matveev, D.A.Shvedov, BINP, Novosibirsk, Russia
}

\begin{abstract}
In this paper we present a project of a Booster-injector for the Duke FEL storage ring. The Booster will provide full energy injection into the storage ring at energy from 0.25 to $1.2 \mathrm{GeV}$. The Duke storage ring FEL (SR FEL) operates in lasing mode with $193-700 \mathrm{~nm}$ wavelength range $[1,2]$. This range will be extended into VUV in near future $[3,4]$. The geometry of the Duke SR FEL provides for head-on collision of e-beam and FEL photons. This mode of operation is used for High Intensity $\gamma$-ray Source (HI $\gamma \mathrm{S}$ facility) generating intense beams of $\gamma$-rays from 1 $\mathrm{MeV}$ to about $200 \mathrm{MeV}$ (currently from $2 \mathrm{MeV}$ to 58 $\mathrm{MeV}$ ). Generation of $\gamma$-rays with energy exceeding 20 $\mathrm{MeV}$ causes the loss of electrons, which will be replaced by new electrons from the Booster, operating in a top-up mode. The Booster has a robust FODO lattice. In this paper we present the design of the Booster and its magnetic elements. For 2D and 3D magnet design of the magnetic elements we used MERMAID 2D/3D code [6].
\end{abstract}

\section{BOOSTER LATTICE AND DESIGN}

The full-energy Booster-Injector is the standard approach for modern accelerator facilities. Boosters are notorious for being robust and reliable injectors into modern storage rings. The technology of booster-injectors (i.e. fast ramping rings) is well developed. The proposed Booster-Injector will provide for effective, full energy top-off injection into $\mathrm{HI} \gamma \mathrm{S}$ facility at operation energies from $0.25 \mathrm{GeV}$ to $1.2 \mathrm{GeV}$ [5]. It will transfer the electron bunches into the Duke storage with the repetition rate required for compensation of the lost electrons. The shortest ramping cycle is about $1.5 \mathrm{sec}$. We will use the existing $250 \mathrm{MeV}$ linac to inject into the Booster. The linac-to-booster transport line will re-use many of the existing vacuum components and magnets

We chose the robust FODO lattice for the booster ring to simplify its operation and to keep its cost modest. The Booster has a race-track shape. Two arcs are separated by $4.5 \mathrm{~m}$ long straight sections used for the RF cavity, the injection and the extraction kickers and septums. Each arc consists of 6 bending magnets with parallel edges, 4 focusing and 3 defocusing quadrupoles. They form a simple, and a very stable FODO lattice. The booster has double bilateral symmetry.

The major cost-saving requirement of fitting the Booster into the existing North-East corner of the storage

*This work is supported by the Dean of Natural Sciences, Duke University
Table 1: Main parameters of the Booster (at $1.2 \mathrm{GeV}$ )

\begin{tabular}{ll} 
Maximum beam energy [GeV] & 1.2 \\
Injection energy $[\mathrm{GeV}]$ & 0.25 \\
Average beam current $[\mathrm{mA}]$ & 100 \\
Circumference $[\mathrm{m}]$ & 28.544 \\
Bending radius $[\mathrm{m}]$ & 2.27 \\
RF frequency $[\mathrm{MHz}]$ & 178.55 \\
Number of bunches & $8-17$ \\
Energy rise rate $[\mathrm{sec}]$ & $1-2$ \\
Beam emittance $\varepsilon_{x}, \varepsilon_{y}[\mathrm{~nm}$ rad] & $330 / 15$ \\
Maximum $\beta_{x} / \beta_{y} / \eta_{x}[\mathrm{~m}]$ & $24.6 / 10.2 / 1.6$ \\
Betatron tunes $Q_{y} / Q_{x}$ & $0.46 / 2.41$ \\
Momentum compaction factor & 0.148 \\
Natural chromaticities $C_{y} / C_{x}$ & $-2.3 /-2.4$ \\
Damping times $\tau_{x, y} / \tau_{s}[\mathrm{mS}]$ & $2.83 / 1.42$ \\
Energy loss per turn $[\mathrm{KeV}]$ & 80.7 \\
\hline
\end{tabular}

Magnetic System:

Dipoles: ea./ $B_{\max }[\mathrm{T}] / L_{\text {eff }}[\mathrm{m}]$

$12 / 1.76 / 1.19$

Quadrupoles:

QF1: $\quad$ ea. $/ G_{\max }[\mathrm{T} / \mathrm{m}] / L_{\text {eff }}[\mathrm{m}]$

$4 / 27.2 / 0.149$

QF2: $\quad$ ea./ $G_{\max }[\mathrm{T} / \mathrm{m}] / L_{\text {eff }}[\mathrm{m}]$

4/ $19.5 / 0.149$

QD1: $\quad$ ea./ $G_{\max }[\mathrm{T} / \mathrm{m}] / L_{\text {eff }}[\mathrm{m}]$

QD2: $\quad$ ea./ $G_{\max }[\mathrm{T} / \mathrm{m}] / L_{\text {eff }}[\mathrm{m}]$

4/ $8.4 / 0.142$

2/ $11.2 / 0.142$

ring room imposes limitations on its structure and parameters. Table 1 lists the major parameters of the Booster. Figure 1 shows its layout. $\beta$-functions and $\eta$ function for half of the ring are shown in Figure 2.

All main booster magnets (the dipoles and the quadrupoles) will be powered in series by one power supply.

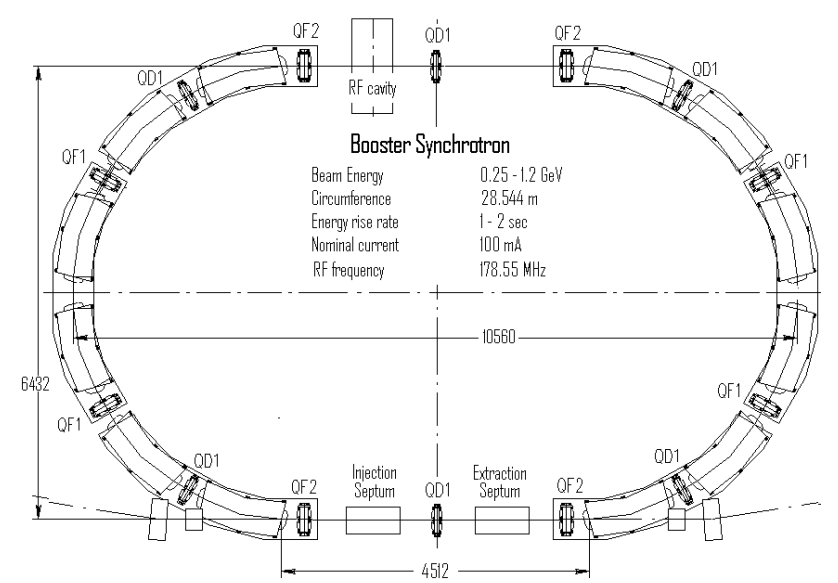

Figure 1: Layout of the Booster-Synchrotron. 


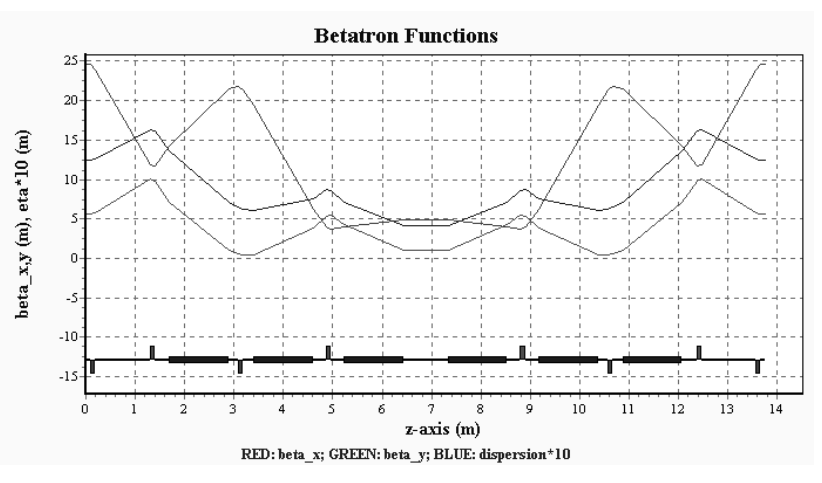

Figure 2: $\beta$ - and $\eta$-functions for half of the Booster ring.

A small number of auxiliary power supplies will be used for trim dipoles and sextupoles. This capability will lead to a convenient and efficient injection into the HI $\gamma \mathrm{S}$ facility. The current of the main power supply will be linearly ramped from the injection to the extraction energy. The current of the main power supply will linearly ramped from the injection to the extraction energy. This approach simplifies the control system. The use of rectangular magnets with edge focusing (the fringe field focusing) provides for partial vertical focusing.

We plan to use a simple vertical single-turn injection. The $250 \mathrm{MeV}$ e-beam from the linac will be turned towards the Booster by a horizontal Lambertson-type septum-magnet. It will be set onto the equilibrium orbit by a kicker located a quarter of betatron oscillation from the septum. The kicker pulse duration will be $95 \mathrm{nsec}$. We also suppose to use vertical extraction scheme. The vertical tune and $\beta$-functions are designed to optimize the efficiency of the extraction kicker (see Figure 2). The RF frequency of the Booster and the storage ring are identical. The RF harmonic numbers are 17 and 64 respectively. This feature allows us transfer individual bunch from a desirable RF bucket of the Booster into selected RF buckets of the storage ring. We will eject up to 17 bunches per ramping cycle into chosen RF buckets of the Duke storage ring. One of the most challenging part of the project will be the ejection kicker with the pulse duration of about 11 nanoseconds. The prototype of such kicker generating about 12 nanoseconds pulses with necessary amplitude was successfully tested.

\section{MAGNETIC SYSTEM AND MAGNET DESIGN}

The parameters of the bending magnets and quadrupoles are listed in Table 1. Figure 3 and 5 show the cross-sections of main magnets. The requirement of fitting the Booster into the existing facility and existing available area causes us to use rather high field of $17.4 \mathrm{kGs}$ (see Table 1) in the bending magnet. This is a challenging task for pulsed dipoles with $\sim 1$ sec ramping time. Rather comprehensive and long end-chamfers reduce dynamic deviation of the effective dipole length (Figure 4). We have experience in developing proto-types of similar magnets for fast ramping boosters. The yokes for both quadrupoles and bending magnets will be fabricated of 1 $\mathrm{mm}$ thick laminated iron. 3D chamfers were also developed for the quadrupoles using MERMAID 2D/3D code [6].

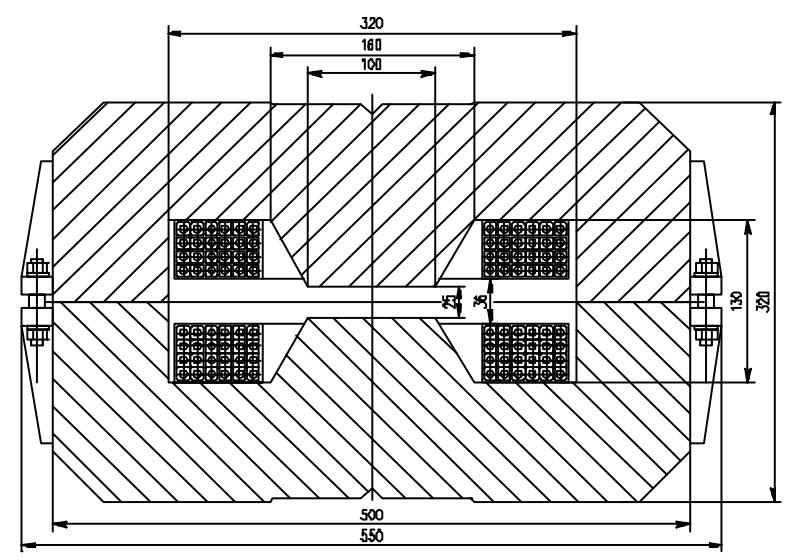

Figure 3: Cross-section of the Booster's bending magnet.

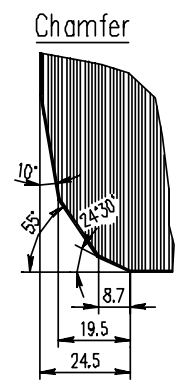

Figure 4: The end chamfer of the dipole magnet.

The vertical gap in the bending magnets of $27 \mathrm{~mm}$ and aperture of quadrupoles of $\mathrm{D}=50 \mathrm{~mm}$ are sufficient for efficient injection and extraction.

We will use four families of quadrupoles in QF1, QF2, QD1 and QD2 with turn ratio of 10, 7, 3 and 4. The above ratios are chosen by the required strengths of quadrupoles fed by the same current.

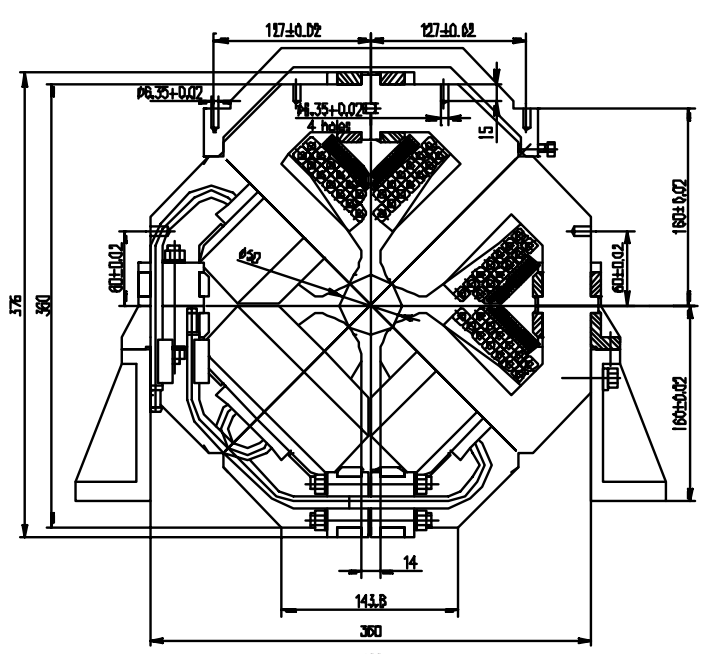

Figure 5: Cross-section of the Booster's quadrupole. 
The saturation affects mostly the large quadrupoles (QF1) and the dipoles. The level of saturation for the dipole and QF1 quadrupole are carefully chosen to minimize the distortion of lattice. At $1.2 \mathrm{GeV}$, the dipole field deviates for the linear dependence for $3.2 \%$. The gradient in QF1 quads has $2.4 \%$ non-linear deviation at the same energy. Meanwhile, the deviations in the QF2, QD1 and QD2 quadrupoles are very small and are at 0.1$0.2 \%$ level. As the results, the betatron tunes deviate during the ramp to $1.2 \mathrm{GeV}$ for $\Delta v \mathrm{v} \approx 0.042$ and $\Delta v y \approx 0.038$. Changes of the tunes are well within the stability region. This allows us to ramp energy without corrections. However, we included the provision for quadrupole corrections as a safety precaution.

\section{RF SYSTEM AND VACUUM CHAMBER}

We will re-use the existing RF system for the Duke storage ring with $40 \mathrm{~kW}$ transmitter to feed the RF cavity of the the Booster. The RF voltage is tunable within 250$600 \mathrm{kV}$ range.

The drawing of the main vacuum chamber of the booster is shown on Figure 6. It has all of the necessary features including the absorbers of synchrotron radiation. The $1-1.5 \mathrm{~mm}$ thickness of the walls is sufficiently transparent to magnetic fields at chosen slow ramping rate.

\section{CONCLUSIONS}

The full-energy robust Booster-injector for the Duke FEL storage ring is based on the proven technical solution. It will provide reliable top-up injection for all conceivable modes of operation. We plan to start detailed design of the Booster in Fall of 2001. We expect to complete the project in 2004.

The use of full energy injection will drastically improve the reliability and the range of parameters of the Duke FEL storage ring light sources ranging for the IR to $\gamma$ rays.

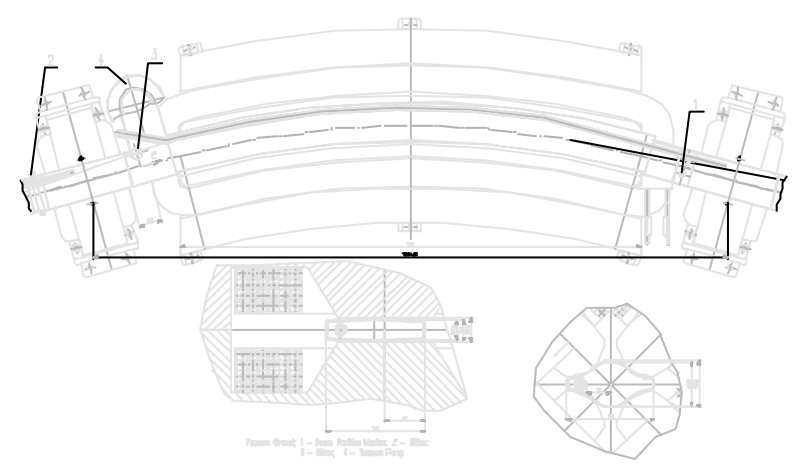

Figure 6: Vacuum chamber of the Booster.

\section{REFERENCES}

1] V.N.Litvinenko, S.H.Park, I.V.Pinayev, Y.Wu, M.Emamian, N.Hower, O.Oakeley, G.Swift, P.Wang, Nucl. Instr. and Meth A429 (1999) 151.

[2] Y.Wu, V.N.Litvinenko, B.Burnham, S.H.Park and J.M.J.Madey, IEEE Transaction on Nuclear Science, Vol. 44, No. 5, October 1997, p.1753.

[3] V.N.Litvinenko, S.H.Park, I.V.Pinayev, Y.Wu, "Operation of the OK-4/Duke storage ring FEL below $200 \mathrm{~nm}$ ", accepted for publication in Proc. of $22^{\text {nd }}$ International FEL Conference, Durham, NC, August 13-18, 2000.

[4] V.N.Litvinenko, O.A.Shevchenko, N.A.Vinokurov, Y.Wu, "Predictions and Expected Performance for the VUV OK-5/Duke Storage Ring FEL with Variable Polarization", accepted for publication in Proc. of $22^{\text {nd }}$ International FEL Conference, Durham, NC, August 13-18, 2000.

[5] HI $\gamma \mathrm{S}$ proposal to the US Department of Energy, Technical Description, September 2000.

[6] Mermaid, the 2D/3D code for magnetic design, (c) A.N.Dubrovin, Novosibirsk, Russia 